\title{
La direzione per chi ha il rene policistico è una sola: si va avanti!
}



Luisa Sternfeld Pavia
Cari Amici,

In questo ultimo periodo sono successe tante cose e tanti sono stati gli eventi che AIRP ha promosso o ai quali ha partecipato: voglio raccontarveli in ordine cronologico e farveli vivere da vicino come se ognuno di voi fosse stato presente.

Cominciamo dal 9 marzo: come sapete questa è la data in cui ricorre la Giornata Mondiale del Rene e quindi, proprio in concomitanza di questo giorno, abbiamo deciso di lanciare la nuova campagna di comunicazione istituzionale di AIRP, una campagna di cui siamo particolarmente orgogliosi e che sta riscuotendo ampissimi consensi da parte di tutti coloro che l'hanno vista. La campagna è sostanzialmente una dichiarazione di impegno improntata al pensiero positivo e alla nostra volontà di fare sempre di più per la causa dei pazienti affetti da rene policistico. II messaggio interpreta, per la scelta tematica e per il modo in cui è stato realizzato, quanto di più profondo anima tutti noi di AIRP ed è per questo che ci sentiamo così ben rappresentati da questo "racconto" che include diversi media: uno spot televisivo di 30 secondi, uno spot per la radio e uscite stampa a pagina intera. Nello spot vediamo una donna che sta facendo jogging lungo una stradina di campagna: la donna corre con passo deciso, lo sguardo rivolto in avanti. La sua corsa, enfatizzata dall'effetto del rallenty, vuole essere una metafora del progresso che la scienza e la medicina stanno compiendo nella lotta contro la complessa patologia del rene policistico. La voce narrante enfatizza questa iconografia con un testo che parla dei grandi passi che la medicina

Accepted: May 2, 2017

Published online: May 25, 2017

Indirizzo per la corrispondenza:

Dr. ssa Luisa Sternfeld Pavia

AIRP Associazione Italiana Rene Policistico onlus

Via A. Bazzini 2

20131 Milano

luisa.sternfeld.airp@renepolicistico.it ha già compiuto e che potrà ancora compiere in futuro, con una frase emblematica che non lascia dubbi sulle finalità e sulla convinzione dell'Associazione su questo tema: "per chi ha il rene policistico c'è una sola direzione: si va avanti!" (https://www.youtube.com/watch?v=syQYhO1Fi3M). Con questa campagna di comunicazione AIRP vuole dare un messaggio di grande incoraggiamento ai Pazienti italiani ma anche ai Medici e ai Ricercatori fortemente impegnati a migliorare le condizioni di vita di chi è affetto da questa patologia, con la speranza che un giorno si possa giungere a una cura risolutiva. Voglio ancora una volta ringraziare di cuore tutti coloro che hanno contribuito alla realizzazione della campagna, per l'impegno e per la partecipazione alla nostra causa!

Ma proseguiamo e passiamo all'11 marzo, data in cui si è tenuta I'Assemblea Ordinaria dei Soci AIRP dedicata all'approvazione del bilancio 2016. Un bilancio sostanzialmente positivo che ci ha consentito di crescere nel numero e nella qualità dei nostri interventi di contributo a diverse ricerche (http://www.renepolicistico.it/bilancio/). La parte del leone nell'Assemblea è stato l'intervento del Prof. Francesco Scolari che ha fatto il punto sulle recenti evoluzioni dell'importantissima questione del Tolvaptan.

Siamo ora giunti al 15-17 marzo: ho partecipato al Congresso di Cardionefrologia 2017 che si è tenuto a Roma all'Hotel NH Villa Carpegna.

L'elevato livello dei relatori, il grande numero di partecipanti, la nutrita presenza di medici provenienti da numerosi Paesi del mondo sono elementi di grande vitalità che testimoniano l'importanza del dibattito scientifico che in questi ultimi anni si sono focalizzati sulle tematiche a noi più vicine. In questo contesto ha avuto un ruolo di grande importanza la sessione dedicata al paziente affetto da ADPKD. La sessione, che ha visto il susseguirsi di quattro importanti relazioni, aveva l'obiettivo di fare il punto su: le complicanze cardiovascolari del paziente con ADPKD, l'ipertensione associata a ADPKD, l'importanza della misurazione e stima del filtrato glomerulare e infine le prospettive terapeutiche dell'ADPKD (Fig. 1).

La prima relazione è stata tenuta dal Prof. Vicente E. Torres (Rochester, USA), che ha illustrato le varie tipologie di problematiche cardiovascolari spesso associate all'ADPKD, e la loro incidenza. A seguire si è tenuta la relazione del Prof. Yannick Le Meur (Brest, Francia), sul 


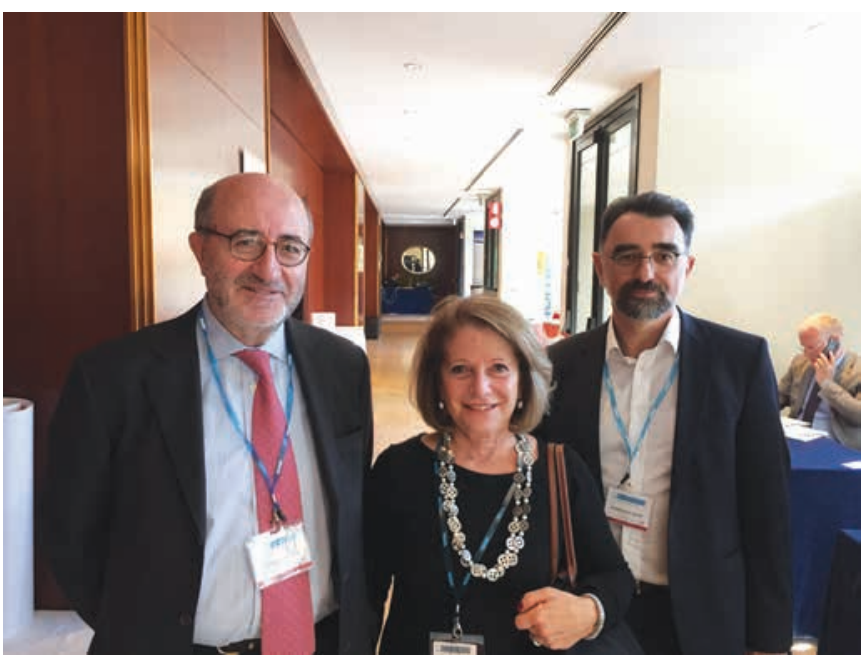

Fig. 1 - Da sinistra: il Prof. Vicente E. Torres, Luisa Sternfeld Pavia e il Prof. Yannick Le Meur al Congresso di Cardionefrologia 2017, tenutosi a Roma dal 15 al 17 marzo 2017.

tema "Ipertensione e ADPKD". La terza relazione è stata tenuta dal Prof. Massimo Cirillo di Salerno, e riguardava l'importanza e le tecniche di stima del filtrato glomerulare ( $v s$ il filtrato misurato). L'ultima relazione della V Sessione è stata presentata dal Prof. Torres. La relazione trattava delle prospettive terapeutiche del paziente con ADPKD. Nelle prossime pagine troverete il report di questa sessione su ADPKD.

L'ultima data di questo excursus è il $\mathbf{2 7}$ marzo: in questa giornata si è svolto a Bologna un incontro tra AIRP e la rete di Associazioni di Bambini con Malattie Renali.

Un incontro molto costruttivo, con il desiderio di tutte le associazioni di portare avanti dei progetti comuni che, se tutti insieme, potranno avere un peso diverso. Intanto stiamo programmando una gita (come quella di Genova dell'anno scorso) a Torino. Appena saprò la data, provvederemo a comunicarvelo.

Pochi giorni più avanti e siamo al 6 aprile. In questa data è avvenuta la cerimonia di consegna di una importante donazione che AIRP ha deciso di devolvere all'UO di Nefrologia, Ospedale Pediatrico Bambino Gesù, IRCCS (Responsabile Prof. Francesco Emma) di Roma, Responsabile del Progetto Dott.ssa Laura Massella, come supporto al progetto "Diagnosi genetica di rene policistico recessivo e dominante: analisi molecolare con tecnica NGS" (Fig. 2).

Eccoci arrivati al $\mathbf{2 2}$ aprile: in questa giornata si è tenuto a San Giovanni Rotondo, presso l'Ospedale "Casa Sollievo della Sofferenza", il primo dei Workshop "Un salotto tutto nostro". Un incontro tra pazienti e i loro familiari per confrontarsi e condividere le diverse esperienze alla presenza anche di un medico specialista, il Dott. Filippo Aucella. In una piacevole e informale atmosfera è stato possibile fare conoscenza tra persone con situazio-

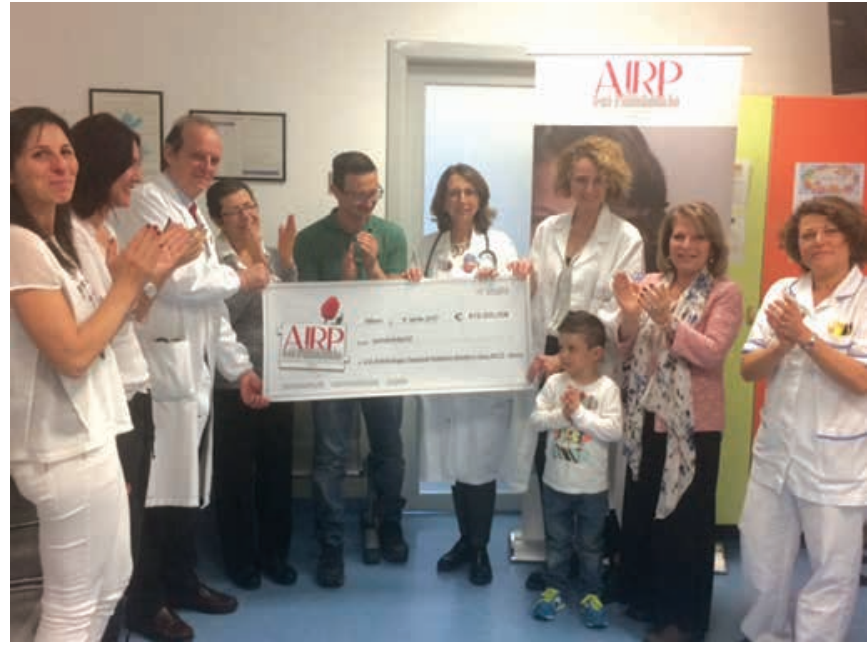

Fig. 2 - La consegna dell'assegno alla Dott.ssa Laura Massella e al Prof. Francesco Emma dell'UO di Nefrologia presso l'Ospedale Pediatrico Bambino Gesù di Roma.

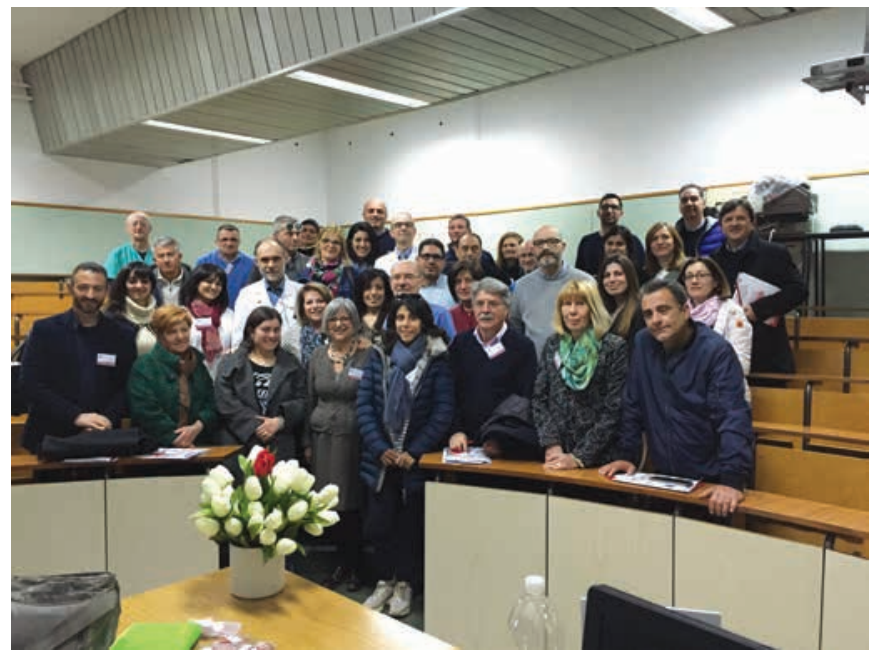

Fig. 3 - Foto di gruppo al primo Workshop di San Giovanni Rotondo, presso l'Ospedale "Casa Sollievo della Sofferenza".

ni simili per condividere i problemi e potersi aiutare uno con l'altro anche solo attraverso lo scambio delle proprie esperienze. La presenza di un medico specialista è stata fondamentale per dare risposte a quesiti e per dare consigli anche pratici per migliorare la vita di tutti i giorni di pazienti e familiari (Fig. 3).

I workshop "Un salotto tutto nostro" proseguiranno durante l'anno in diverse altre località, sempre alla presenza del nefrologo responsabile dell'ambulatorio locale dedicato al Rene Policistico. Per il momento sono già fissate le date del 27 maggio a Catania all'Hotel NH Catania Parco degli Aragonesi, presenti i Dottori Santina Castel- 


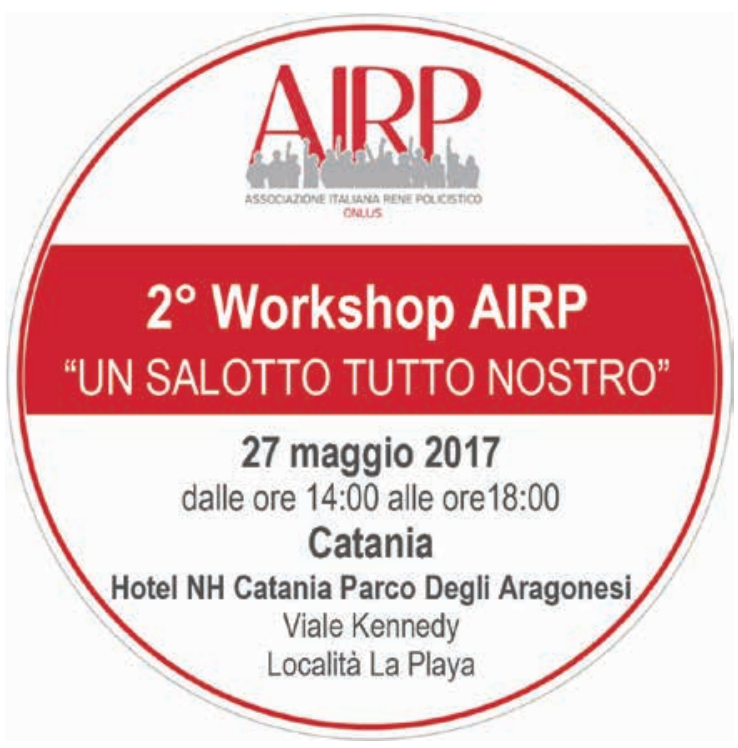

Fig. 4 - Il logo del secondo Workshop AIRP.

lino, Angelo Ferrantelli, Carmelita Marcantoni, Mimmo Santoro (Fig. 4).

A seguire il terzo Workshop a Torino il 16 settembre, al Circolo dei Lettori, in una splendida sala che farà da cornice. Saranno presenti la Dott.ssa Silvana Savoldi e il Prof. Francesco Scolari (Fig. 5).

Cari Amici, ripercorrendo le settimane appena passate ci rendiamo conto di quante attività abbiamo svolto, ma siamo anche consapevoli che le cose che si potrebbero fare sarebbero anche molte di più. Naturalmente, lo sa-

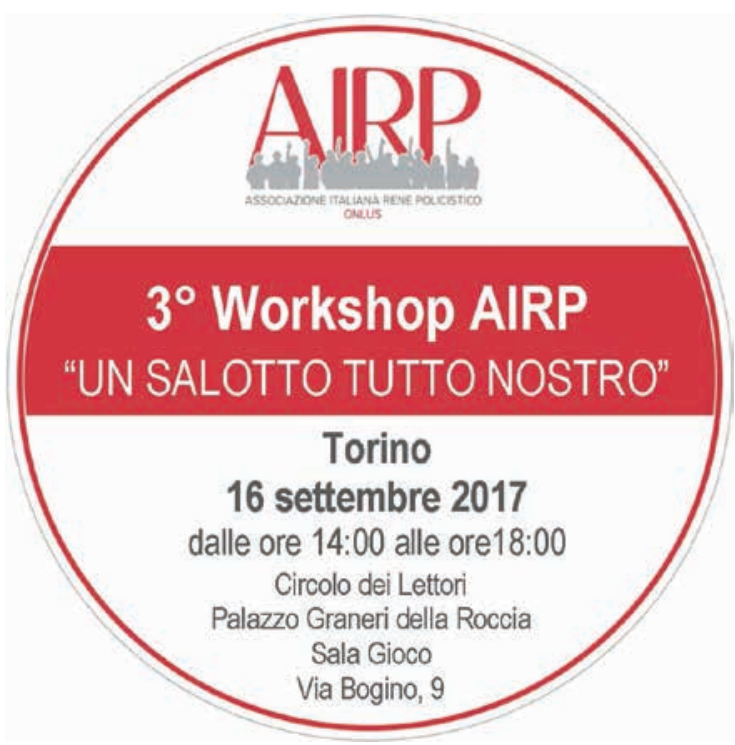

Fig. 5 - Il logo del terzo Workshop AIRP.

pete, noi ce la mettiamo tutta e cerchiamo di distribuire il nostro tempo e le risorse disponibili meglio che possiamo. Ma come dice la nuova campagna di comunicazione AIRP "c'è una sola direzione: si va avanti!". Ed è quello che, con il vostro aiuto, facciamo giorno per giorno.

Un forte abbraccio,
Luisa Sternfeld Pavia Presidente AIRP 